\title{
REGISTERING UPPER ATMOSPHERE PARAMETERS IN EAST SIBERIA WITH FABRY-PEROT INTERFEROMETER KEO SCIENTIFIC “ARINAE”
}

\author{
R.V. Vasilyev \\ Institute of Solar-Terrestrial Physics SB RAS, \\ Irkutsk, Russia,roman_vasilyev@iszf.irk.ru
}

\section{M.F. Artamonov}

Institute of Solar-Terrestrial Physics SB RAS, Irkutsk, Russia, artamonov.maksim@iszf.irk.ru

\section{A.B. Beletsky}

Institute of Solar-Terrestrial Physics SB RAS, Irkutsk, Russia, beletsky@iszf.irk.ru

\section{G.A. Zherebtsov}

Institute of Solar-Terrestrial Physics SB RAS, Irkutsk,Russia,gaz@iszf.irk.ru

\section{I.V. Medvedeva}

Institute of Solar-Terrestrial Physics SB RAS, Irkutsk,Russia,ivmed@iszf.irk.ru

\section{A.V. Mikhalev}

Institute of Solar-Terrestrial Physics SB RAS, Irkutsk,Russia,mikhalev@iszf.irk.ru

\section{T.E. Syrenova}

Institute of Solar-Terrestrial Physics SB RAS, Irkutsk, Russia, angata@iszf.irk.ru
Abstract. We describe the Fabry-Perot interferometer designed to study Earth's upper atmosphere. We propose a modification of the existing data processing method for determining the Doppler shift and Doppler widening and also for separating the observed line intensity and the background intensity. The temperature and wind velocity derived from these parameters are compared with physical characteristics obtained from modeling (NRLMSISE-00, HWM14). We demonstrate that the temperature is determined from the oxygen 630 $\mathrm{nm}$ line irrespective of the hydroxyl signal existing in interference patterns. We show that the interferometer can obtain temperature from the oxygen $557.7 \mathrm{~nm}$ line in case of additional calibration of the device. The observed wind velocity mainly agrees with model data. Night variations in the red and green oxygen lines quite well coincide with those in intensities obtained by devices installed nearby the interferometer.

Keywords: Fabry-Perot interferometry, airglow, upper atmosphere wind, upper atmosphere temperature.

\section{INTRODUCTION}

Spectrophotometric studies of night airglow are one of the main instruments for studying Earth's upper atmosphere [Shefov et al., 2006]. Features of the generation of optical radiation in the upper atmosphere, namely its spectral lines and vertical stratification of airglow for certain wavelengths, provide information on motion and temperature of air masses in various atmospheric layers. A physical phenomenon that makes it possible to determine atmospheric gas velocity and temperature is the Doppler shift of the wavelength of recorded radiation, which arises from collective (wind) or chaotic (temperature) motion of radiating particles. Using wellknown expressions (for example [Landau, Lifshitz, 1988])

$$
\begin{aligned}
& \frac{\lambda_{\mathrm{c}}}{\lambda_{0}}=\left(1-\frac{v}{c}\right), \\
& \frac{\Delta \lambda}{\lambda_{0}}=\sqrt{\frac{k T}{m c^{2}}}
\end{aligned}
$$

( $\lambda_{0}$ is the wavelength of immobile emitting matter, $\lambda_{s}$ is the central wavelength of the registered spectral line, $\Delta \lambda$ is the recorded widening of the spectral line, $v, T$, $m$ are the velocity, temperature, and mass of luminous matter particles, $k$ is the Boltzmann constant, $c$ is the speed of light in vacuum), we can estimate the sensitivity necessary for the successful application of the method:

$$
\begin{aligned}
& \delta \lambda=\lambda_{0}-\lambda_{\mathrm{c}}=\frac{\lambda_{0} v}{c}, \\
& \delta \Delta \lambda=\Delta \lambda_{2}-\Delta \lambda_{1}=\lambda_{0}\left(\sqrt{\frac{k T_{2}}{m c^{2}}}-\sqrt{\frac{k T_{1}}{m c^{2}}}\right) .
\end{aligned}
$$

In order for the instrument to record changes in wind velocity at $10 \mathrm{~m} / \mathrm{s}$ and temperature at $10 \mathrm{~K}$ for a wavelength of $630 \mathrm{~nm}$, its sensitivity should be such that an investigator can observe changes of position and width of the spectral line at $10^{-5}$ and $10^{-4} \mathrm{~nm}$ respectively.

One of the currently available methods for recording the spectral composition of optical radiation with this sensitivity is a method for observing interference in parallel rays in the Fabry-Perot interferometer [Born, Wolf 1973]. There are many scientific instruments (for example, [Wu et al., 2004; Shiokawa et al., 2012; Anderson et al., 2009; Ignatiev et al., 1998]) that use this method to study optical airglow. The field of view of these instruments, except for that described in [Anderson et al., 2009], is units of degrees, so in a single observation session we can obtain characteristics of only a certain local region of the celestial sphere. Therefore, to extend 
functionalities, some of these instruments scan the celestial sphere with an automated periscope as an input window. Interferometers record observations with CCD cameras, which allow one to store an entire interference pattern in digital form and to process measurements after an observation session. A key issue in observations at this sensitivity level is the stability of parameters of an observation system. Modern Fabry-Perot interferometers for observing Earth's upper atmosphere are equipped with temperature stabilization systems and laser calibration light sources adopted to monitor stability of their operation.

We deal with one of such instruments installed in the Geophysical Observatory (GO) of the Institute of SolarTerrestrial Physics SB RAS (the village of Tory, $52^{\circ} \mathrm{N}$, $\left.103^{\circ} \mathrm{E}\right)$. We describe its design and a data processing method. Diurnal variations in some characteristics of the upper atmosphere, obtained with the interferometer, are given and compared to similar parameters recorded with other instruments. A comparison is made with upper atmosphere parameters obtained by models NRLMSISE-00 [Picone et al., 2002] and HWM14 [Drob et al., 2015]. We have used observational data acquired at ISTP SB RAS GO from June 2016 to February 2017.

\section{INTERFEROMETER DESIGN}

The Fabry-Perot interferometer installed in GO is manufactured by KEO Scientific (Canada) and is called KEO Scientific Arinae. A detailed description of its design is given in [Shiokawa et al., 2012]. We present only some distinctive features essential for our study. The schematic of the tool is shown in Figure 1 (left). One of the main specific systems of the instrument is the periscope consisting of two mirrors, which can independently rotate in perpendicular planes with the aid of step motors (Figure 1, right). The step motors are controlled automatically, thereby providing the opportunity to adaptively manage the observation process and to aim the periscope to any point in the celestial sphere. After the input window, the radiation passes through a passband filter $70 \mathrm{~mm}$ in diameter. The diameter of the filter determines the diameter of the pupil of the aperture of the instrument. The interferometer is equipped with a set of six equal-sized filters with a passband of $\sim 1 \mathrm{~nm}$ each and central wavelengths of the passband of $630.0,557.7,427.8,589.3,732.0$, and $843.0 \mathrm{~nm}$. The filters are placed in a filter wheel cassette. Like the input window, the filter wheel cassette is controlled by a step motor with the ability to automatically control and change the filters according to a preset program. The cassette is equipped with a temperature stabilization system that automatically maintains the desired constant temperature of the filters. After the filter, the light flux comes to the Fabry-Perot etalon, made in the form of two circular reflecting surfaces $70 \mathrm{~mm}$ in diameter with an air gap $15 \mathrm{~mm}$ high.

The air gap is hermetically sealed off from the environment. The reflecting surfaces are made of a material with a low coefficient of thermal expansion $\left(0 \pm 0.007 \cdot 10^{-6} \mathrm{~K}^{-1}\right.$ in a range $\left.0-50{ }^{\circ} \mathrm{C}\right)$ and are coated with a specialized reflective composition optimized for working with radiation at wavelengths of 630.0 and $557.7 \mathrm{~nm}$ (the reflection coefficient is $0.7-0.9$ ). The plate surface quality is $\lambda / 100$. The interference pattern is
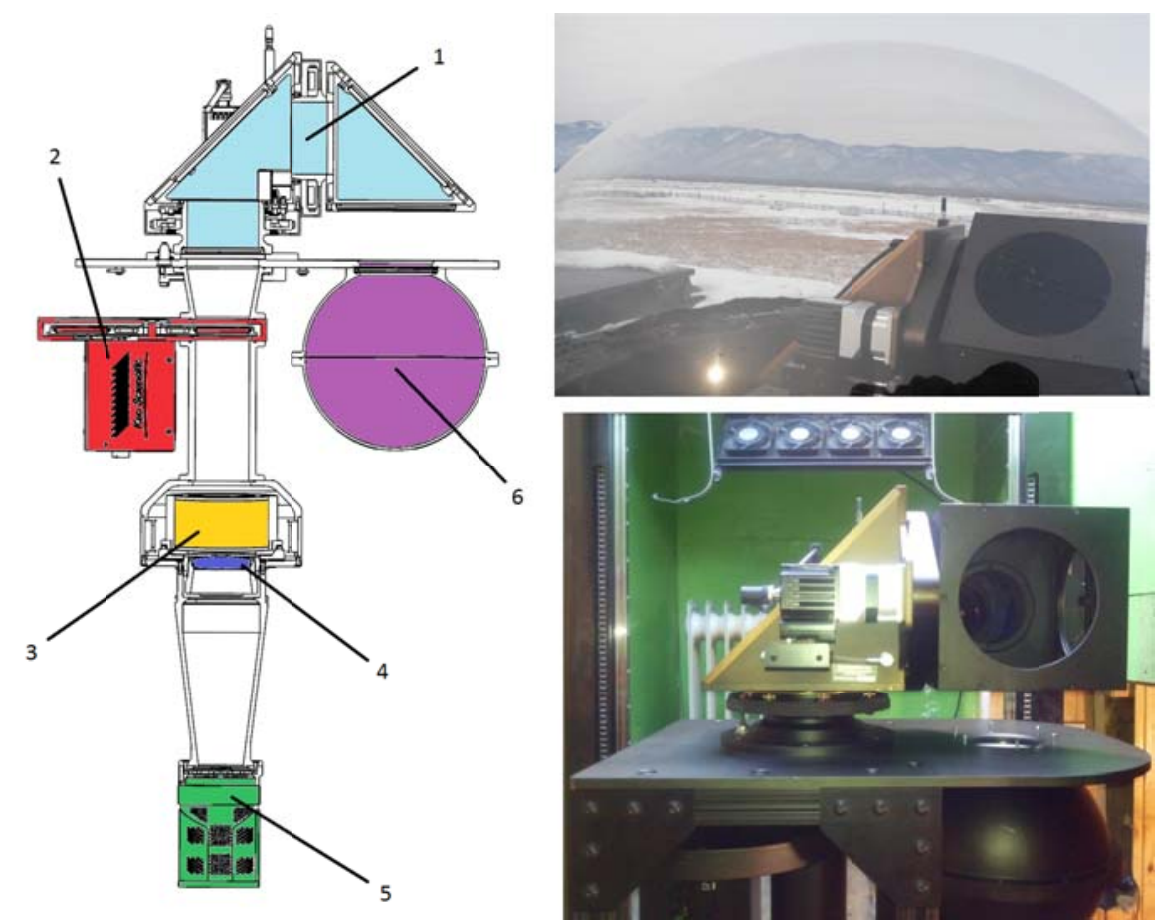

Figure 1. General scheme and photographs of the interferometer periscope: 1 is the periscope; 2 is the filter wheel cassette; 3 is the Fabry-Perot etalon; 4 is the focusing lens; 5 is the CCD camera; 6 is the calibration light-scattering sphere. The input window is oriented such that the interferometer can record a helium-neon laser light scattered in the sphere 
wheel cassette, has a temperature stabilization system that automatically maintains temperature required for operation. The interference pattern, formed on the lower plate of the etalon, is focused by the collecting lens on the CCD matrix. The focal length is about $30 \mathrm{~cm}$; the size of the matrix is $1.3 \times 1.3 \mathrm{~cm}^{2}$. Thus, the field of view of the instrument is $\sim 2.5^{\circ}$. This instrument uses a PIXIS 1024B CCD camera. Resolution of the CCD matrix is $1024 \times 1024$ pixels; to reduce noise, the matrix is cooled to the operating temperature of $-70{ }^{\circ} \mathrm{C}$, using a Peltier element. At this stage of the study, the said resolution is not required; therefore, to reduce costs for processing information coming from the camera and increase the sensitivity, the image from the camera undergoes the socalled binning procedure. The actual resolution decreases to $512 \times 512$ pixels. For additional calibration, the interferometer is equipped with a helium-neon laser connected through a light guide to a special lightscattering sphere. The output window of the sphere is attached to the periscope support. The input window can be oriented such that laser light from the scattering sphere would fall on the input window of the interferometer and can automatically calibrate the instrument during measurements. The output of the laser is equipped with an automatically controlled shutter to prevent laser light from entering the input window of the interferometer when observing the celestial sphere. The instrument is controlled by a personal computer
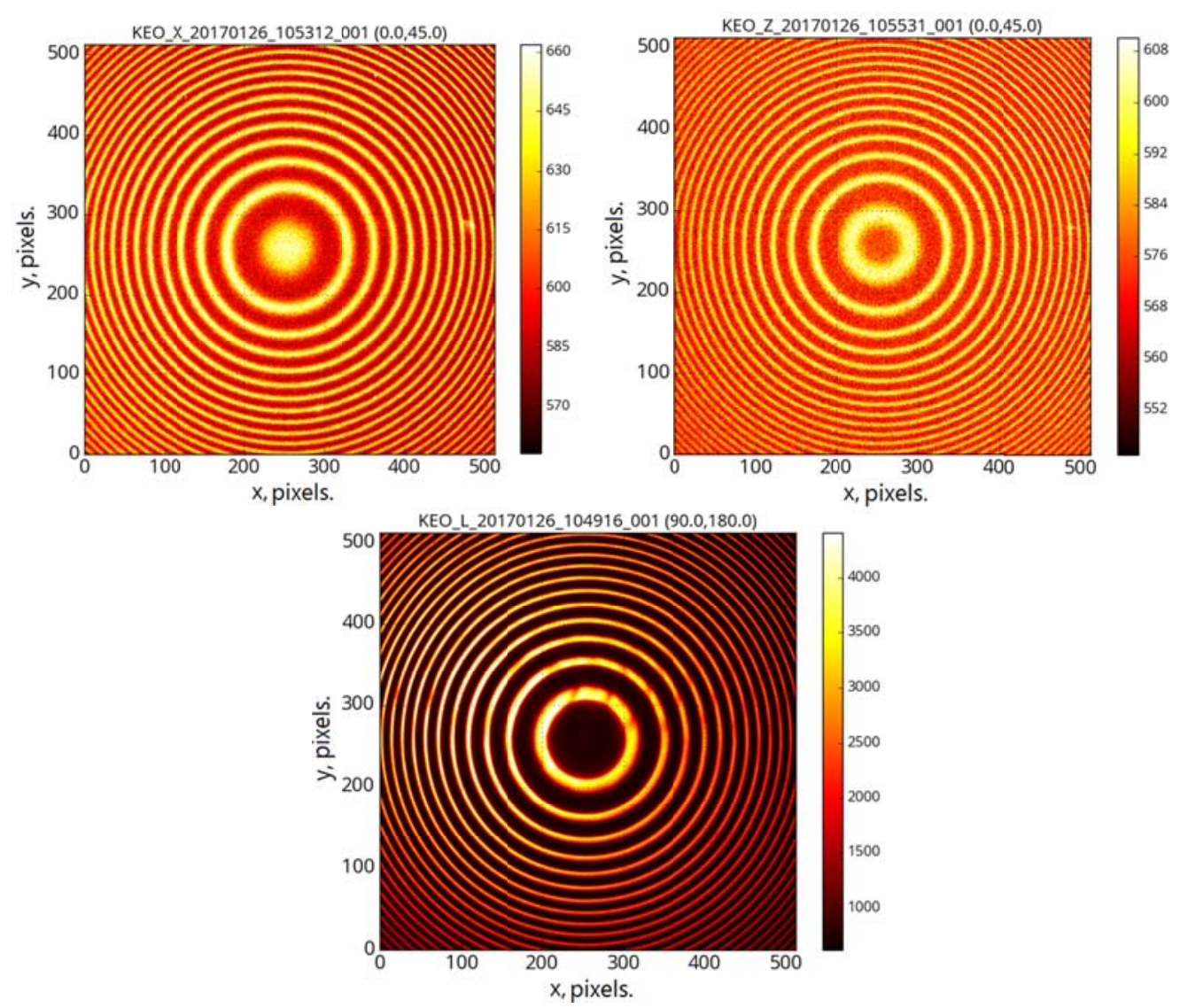

Figure 2. Interferograms obtained from observation of nightglow at a wavelength of $630.0 \mathrm{~nm}$ (top left) and $557.7 \mathrm{~nm}$ (top right). At the bottom is an interferogram from observation of the helium-neon laser light $(632.8 \mathrm{~nm})$. The color intensity scale is in relative units 
[Ignatyev, Yugov, 1995]); however, the use of twodimensional CCD matrices for recording interference patterns and the computational capabilities of modern computers open up somewhat greater possibilities of image processing. Therefore, the basis for processing observational data is the method described in [Harding et al., 2014]. Its application requires us to represent a two-dimensional interferogram as one-dimensional through circular integration of the available twodimensional dataset, using the method described in [Makela et al., 2011]. As a result, the two-dimensional interferogram is represented as one-dimensional, reflecting the dependence of intensity on distance, which is measured from the center of the interference pattern. For successful circular integration of the interferograms obtained from airglow observation (especially under variable intensity of recorded light), we should periodically register calibration laser interferograms. These calibration data help to determine current parameters of the measuring system, such as the center of the interference pattern and the instrumental function. The instrumental function, which, in fact, is a modified Airy function [Borne, Wolff, 1973], contains additional information on inhomogeneities of the optical system of the interferometer. Hereinafter, we utilize this instrumental function to describe interferograms obtained from nightglow observations. This model is an integral function composed of a set of modified Airy functions entering the integral with values of intensity and wavelength corresponding to an assumed form of spectral line of detected light. Obviously, the determination of parameters of the instrumental function and the model function describing a recorded signal requires performing the procedure of "fitting" the model functions to experimental data. For this purpose, we use least-squares minimization with the Levenberg-Marquardt algorithm [Marquardt, 1963].

Harding et al. [2014] logically present moments of reduction of the Airy function describing the interference pattern from monochromatic radiation at the output of the ideal Fabry-Perot etalon to a modified form; therefore, it makes no sense to dwell on these operations here. We give only the final result and describe some modifications essential for this study. The modified Airy function has the form

$$
\bar{A}(r, \lambda)=\int_{0}^{r_{\mathrm{m}}} b(s, r) A(s, \lambda) d s+B,
$$

where $\lambda$ is the radiation wavelength, $r$ is the distance from the center of the interference pattern, $r_{\mathrm{m}}$ is the radius of the boundary of the interference pattern. In fact, (5) is the convolution of the Airy function, which has a variable intensity depending on $r$, necessary to take into account vignetting of the optical system,

$$
A(s, \lambda)=\frac{I_{0}\left(1+I_{1} \frac{s}{r_{\mathrm{m}}}+I_{2} \frac{s^{2}}{r_{\mathrm{m}}^{2}}\right)}{1+\frac{4 R}{(1-R)^{2}} \sin ^{2}\left(\frac{2 \pi n t}{\lambda} \cos \alpha s\right)}
$$

with a kernel reflecting defects of the optical system, which blur the image on the matrix,

$$
b(s, r)=\frac{1}{\sqrt{2 \pi \sigma^{2}(r)}} \exp \left\{\frac{(s-r)^{2}}{\sigma^{2}(r)}\right\}
$$

plus a background $B$, which naturally arises from thermal noises of pixels and penetration of the background radiation, uniformly distributed over wavelengths, through the input filter and etalon.

In the above expressions, $R$ is the reflection coefficient of the Fabry-Perot etalon's surfaces, $n$ is the refractive index of the medium between the reflecting surfaces of the etalon, $t$ is the distance between the etalon's surfaces, $\alpha$ is the magnification factor; $I_{0}, I_{1}, I_{2}$ are constant coefficients.

The dependence of the Gaussian kernel width on the distance in the CCD matrix is as follows:

$$
\sigma(r)=\left(\sigma_{0}+\sigma_{1} \sin \left(\pi \frac{r}{r_{\mathrm{m}}}\right)+\sigma_{2} \cos \left(\pi \frac{r}{r_{\mathrm{m}}}\right)\right),
$$

where $\sigma_{0}, \sigma_{1}, \sigma_{2}$ are constant coefficients.

Expression (5) in [Harding et al., 2014] is used as an instrumental function. By minimizing the difference of this model with one-dimensional laser interferograms, we can find current values of the constant coefficients in (6)-(8), reflection coefficient, gap of the etalon, and magnification factor. The values derived from the minimization are used as nonvarying parameters in the model of night-sky interferograms:

$$
S(r)=\int_{\lambda_{1}}^{\lambda_{2}} \bar{A}(r, \lambda) Y(\lambda) d \lambda+B,
$$

where $Y(\lambda)$ reflects the shape of the spectral line:

$$
Y(\lambda)=Y_{0}+Y_{1} \exp \left\{-\frac{1}{2}\left(\frac{\lambda-\lambda_{c}}{\Delta \lambda}\right)^{2}\right\} .
$$

Integration limits in (9) are chosen based on characteristics of the input filter. The values reflecting the background $B$, line intensity $I_{0}$, its position $\lambda_{\mathrm{s}}$ and width $\Delta \lambda$ are determined by minimizing the difference between observed data and the model of night-sky interferograms. After minimizing (9) with night-sky observations, $\lambda_{\mathrm{s}}$ and $\Delta \lambda$ obtained from (1) and (2) determine temperature and velocity of the emitting medium along the line of sight of the interferometer.

To improve the stability of the interferogram processing method put forward in [Harding et al., 2014], we modify the expressions describing interference patterns. The following changes in models of calibration laser light and airglow can be considered essential:

1) substituting the polynomial dependence of intensity (the numerator in (6)) by the first terms of the Fourier expansion such that this dependence looks like expression for Gaussian kernel width (8);

2) replacing the magnification factor $\alpha$ by the direct calculation of the inclination angle of input radiation 
registered in a certain coordinate of the $\mathrm{CCD}$ matrix $r$ : $\alpha r=\operatorname{arctg}(r / F)$;

3) introducing the squared relationship between the background and the distance on the CCD matrix $B=B_{0}+$ $B_{1} r^{2}$

4) symmetric expansion of a one-dimensional interferogram into the negative scale $r$.

The last operation doubles the number of points in the existing dataset. At first glance, this leads only to a useless increase in the computational time for minimization. However, we think that this approach is valid since it facilitates more stable operation of the minimization algorithm. In particular, it automatically meets the natural requirement of the zero derivative for the intensity at the center of an interference pattern. Examples of one-

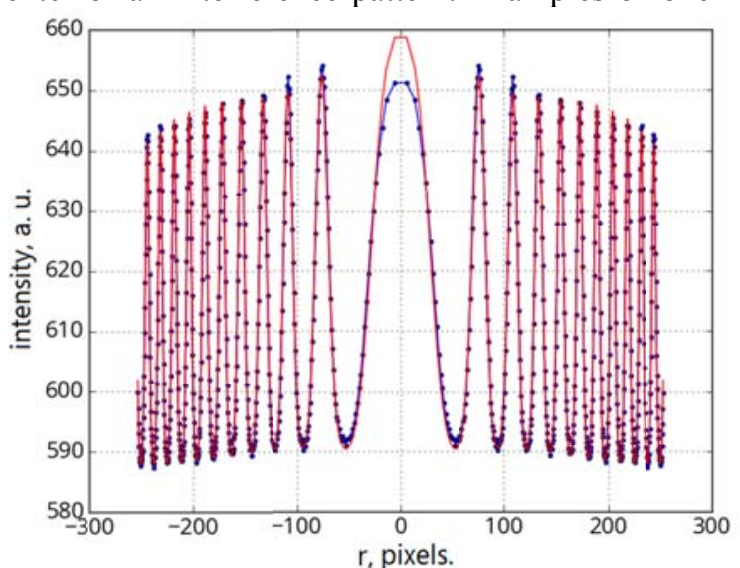

dimensional interferograms and results of minimization of the models to the observational data used in this paper are given in Figure 3. As seen below, despite some deviations of the resulting model functions from experimental data, the reliability of the obtained upper atmosphere parameters is at an acceptable level.

It should be noted that the interferogram data processing methods and minimization procedures are performed using the Python language [van Rossum, 1995], as well as packages Mahotas [Coelho, 2013] for image processing to form one-dimensional interferograms and Lmfit [Newville et al., 2014] to implement procedures for fitting model functions to observational results.
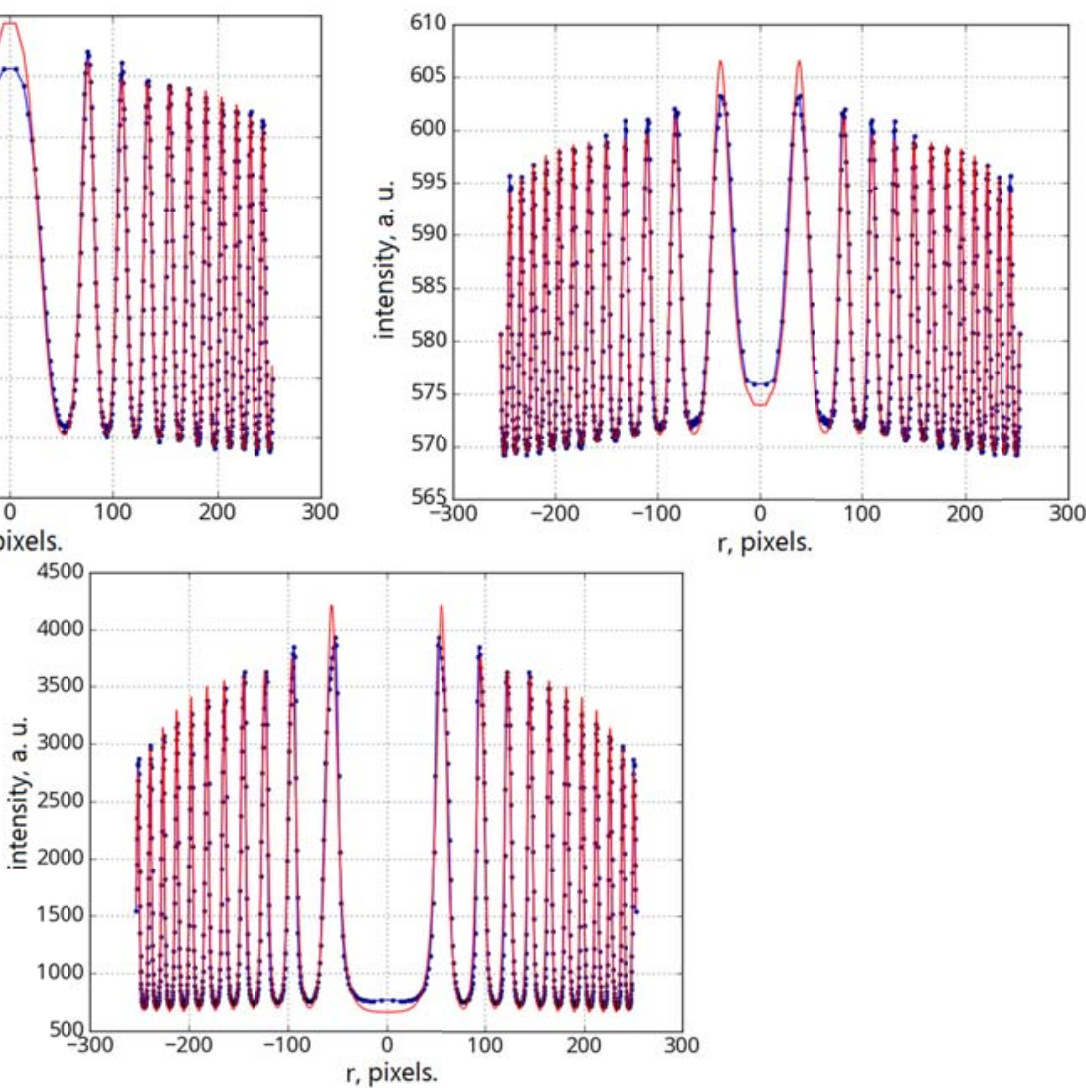

Figure 3. One-dimensional interferograms (blue curves) and functions with selected parameters describing these interferograms (red curves). Interferograms derived from observation of the nightglow at a wavelength of $630.0 \mathrm{~nm}$ (top left) and 557.7 $\mathrm{nm}$ (top right). At the bottom is an interferogram obtained from observation of helium-neon laser light $(632.8 \mathrm{~nm})$

The mentioned capability of the interferometer to automatically change the direction of observation along with the assumption of a certain time of medium stationarity in an observed region determines the method of wind velocity measurements. Suppose that at heights corresponding to the maximum intensity of atomic oxygen airglow in a region with a characteristic size of several hundreds of kilometers the wind blows in the same direction for 15 minutes. Then, if during this time we perform observations in the north, south, west, and east directions at an angle to the horizon, the obtained interferograms would be formed by radiation whose wavelength is shifted by some value depending on the direction of observation. This shift is caused by the so-called line-of-sight velocity - the projection of the wind velocity on the direction of observation, combined with a possible systematic shift arising from inaccuracy of the model describing the interferogram. Because of the stationarity of the wind velocity during a series of observations in different directions, the estimated line-of-sight velocities are rigidly bound (Figure 4):

$$
\begin{aligned}
& L_{\mathrm{N}}=L_{0}+H \sin \theta+V \cos \theta, \\
& L_{\mathrm{S}}=L_{0}+H \sin \theta-V \cos \theta, \\
& L_{\mathrm{E}}=L_{0}+H \sin \theta+U \cos \theta, \\
& L_{\mathrm{W}}=L_{0}+H \sin \theta-U \cos \theta,
\end{aligned}
$$




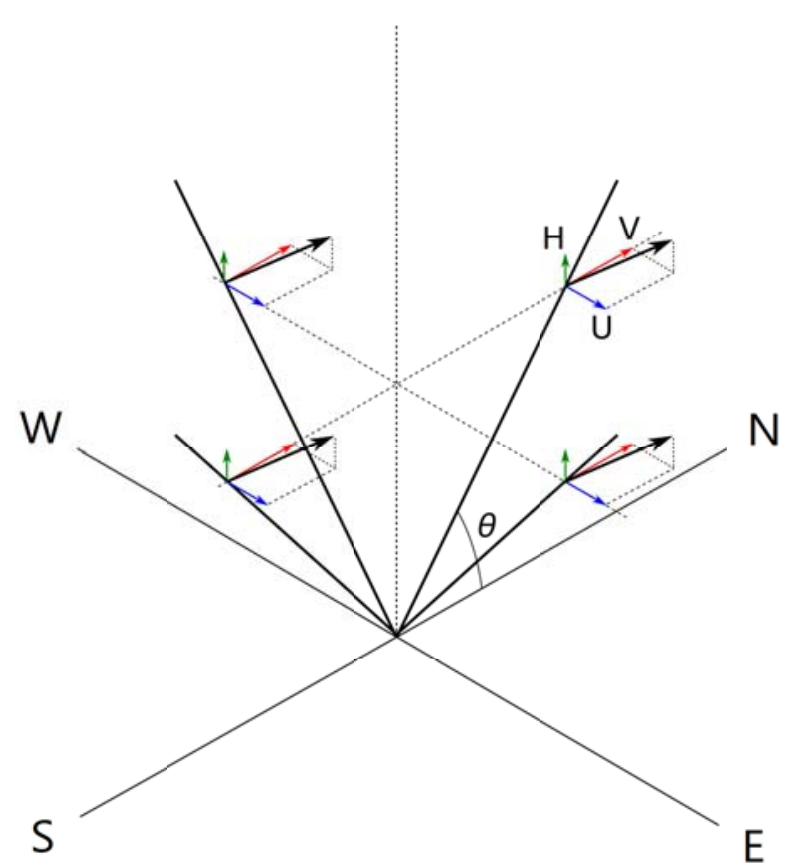

Figure 4. Directions of observations and projection of wind velocity. The black arrow is the full wind velocity, the green arrow is the vertical wind velocity, the red and blue arrows are meridional and zonal components of the horizontal wind velocity

where $L_{\mathrm{N}}, L_{\mathrm{S}}, L_{\mathrm{E}}, L_{\mathrm{W}}$ are line-of-sight velocities in the directions to the north, south, east, and west respectively; $L_{0}$ is the possible systematic shift due to inaccuracy of the model describing the interferogram; $H$ is the vertical wind velocity; $U, V$ are zonal and meridional components of the horizontal wind velocity; $\theta$ is the elevation angle of observation. Expressions (11)-(14) show that to derive values of the zonal and meridional wind, we should subtract line-of-sight velocities of opposite directions:

$$
\begin{aligned}
& L_{\mathrm{N}}-L_{\mathrm{S}}=2 V \cos \theta, \\
& L_{\mathrm{E}}-L_{\mathrm{W}}=2 U \cos \theta .
\end{aligned}
$$

This operation also eliminates the possible systematic error in the measuring system and the vertical wind effect.

Temperature as a scalar quantity does not require a change of direction to be estimated. Of course, there also may be some systematic errors in this parameter in the model, but, unfortunately, a change in directions in this case cannot eliminate them because the stationarity of temperature in time in the observed region does not imply the absence of spatial temperature gradients. Therefore, there are no special requirements for temperature measurements, except for the sufficient exposure time to form an interference pattern.

Thus, the sequence of nighttime observations looks like a set of directions changing with time (see Table).

Typical interferograms for the said exposure times have already been presented in Figures 2, 3. Figure 5 shows variations in the $I_{0}$ line intensity, background optical radiation near the $630 \mathrm{~nm}$ emission $B_{0}$, horizontal wind temperature and speed for the night of January 26-27, 2017, obtained with the described method of Sequence of nighttime observations performed with the inter- ferometer

\begin{tabular}{|l|r|r|}
\hline \multicolumn{1}{|c|}{ Direction } & $\begin{array}{r}\text { Wave- } \\
\text { length }\end{array}$ & \multicolumn{1}{c|}{ Exposition } \\
\hline North & 630.0 & $120 \mathrm{~s}$ \\
\hline North & 557.7 & $60 \mathrm{~s}$ \\
\hline East & 630.0 & $120 \mathrm{~s}$ \\
\hline East & 557.7 & $60 \mathrm{~s}$ \\
\hline South & 630.0 & $120 \mathrm{~s}$ \\
\hline South & 557.7 & $60 \mathrm{~s}$ \\
\hline West & 630.0 & $120 \mathrm{~s}$ \\
\hline West & 557.7 & $60 \mathrm{~s}$ \\
\hline zenith & 630.0 & $120 \mathrm{~s}$ \\
\hline zenith & 557.7 & $60 \mathrm{~s}$ \\
\hline laser & 630.0 & $30 \mathrm{~s}$ \\
\hline
\end{tabular}

observation and processing of the results. We have chosen January 26, 2017 because at this very night in particular observation hours there were abnormally low temperatures $(\sim 200-300 \mathrm{~K})$ estimated from the $630 \mathrm{~nm}$ emission $\left(T_{630}\right)$. Note here that the minimum monthly averages of $T_{630}$ for winter months are $\sim 400-450 \mathrm{~K}$; for summer months, $\sim 600-750 \mathrm{~K}$. The maximum values of $T_{630}$ are recorded in twilight periods of observations: $\sim 800-900 \mathrm{~K}$ for summer and $\sim 700 \mathrm{~K}$ for winter. Figure 6 demonstrates the behavior of the parameters of the instrumental function model, which are the most critical for measuring wind velocity (the product of the thickness of the gap of the etalon and the refractive index $n t$, the focal length $F$ ), and temperature (reflection coefficient of the etalon's surfaces $R$ and the width of the Gaussian kernel describing image blurring $S_{0}$ ). These parameters were obtained from periodic calibration of the tool during the night. The lack of agreement in the behavior of constants of the instrument and characteristics of the recorded light suggests that the model is most likely to be valid and can be used for follow-up studies.

The behavior of the recorded intensity of airglow, temperature, and wind is considered below. Here, we want to discuss the behavior of the background optical radiation. As seen, in the second half of the night for most analyzed directions the mean background value is higher and its variations are considerable. This is due to the presence of an additional light source with a wide spectrum in the field of view of the tool. Such an additional source of light in this spectral range can be the airglow continuum, light from stars, planets, the Moon, and other natural and artificial sources. If there are clouds in the interferometer field of view, the intensity of this additional light source will increase and vary considerably with time. Thus, the parameter $B_{0}$ can be used to indirectly estimate the presence of cloudiness during observations. In this case, the behavior of this parameter means that from the beginning of the observation session on January 26, 2017 at $\sim 18: 00$, the sky was clear, and clouds gathered at $\sim 00: 00$ on January 27 , 2017. This is confirmed by photos taken by wide-angle surveillance cameras installed nearby the interferometer. Despite the appearance of cloudiness, the degree of cloud cover is such that it practically does not affect the behavior of the observed intensity. It is impossible to 

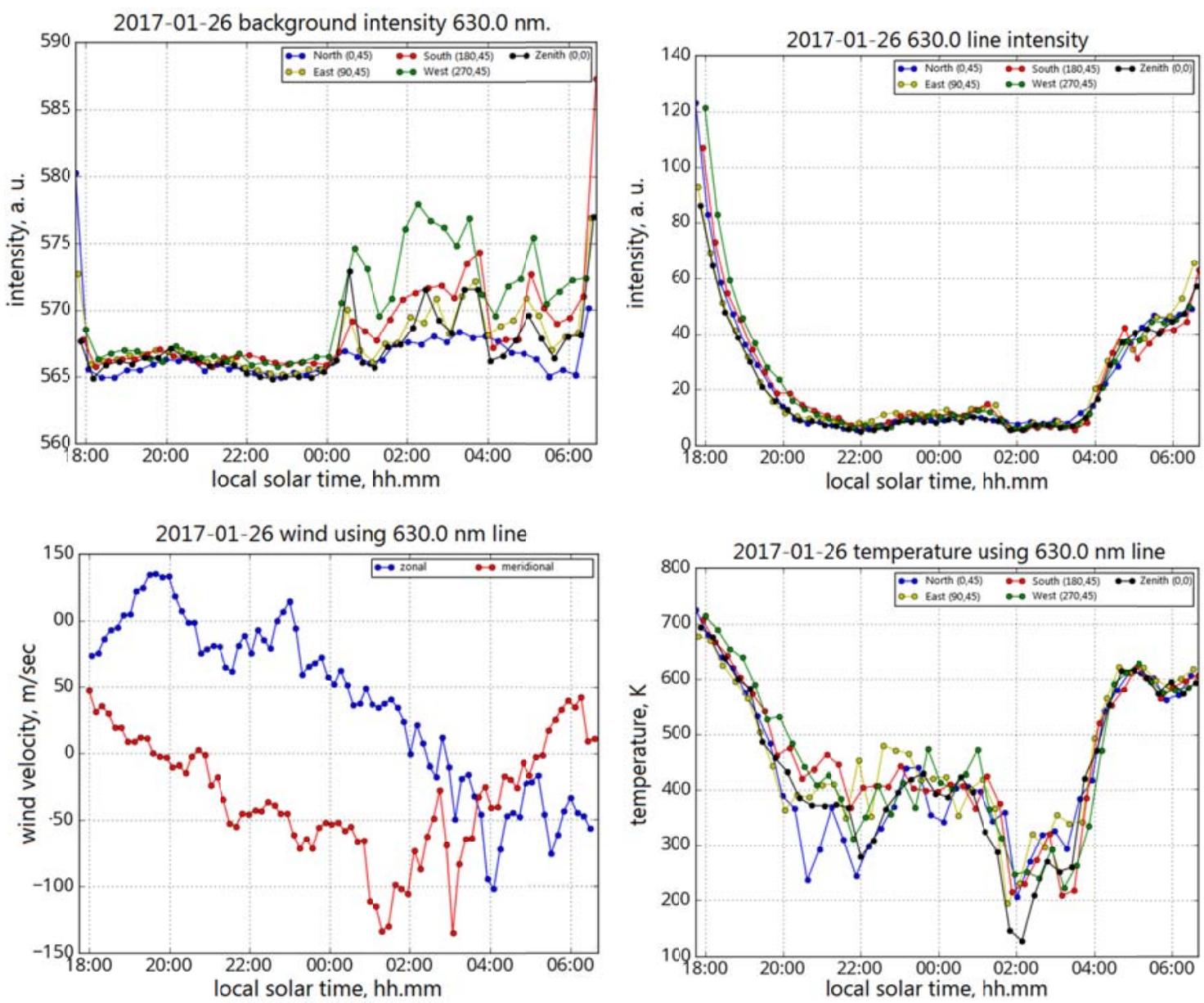

Figure 5. Background intensity (top left), line intensity (top right), zonal and meridional wind velosities (positive directions of wind velocity to the north and east) (bottom left), temperature (bottom right), as observed in the atomic oxygen airglow at $630.0 \mathrm{~nm}$ during the night on January 26-27, 2017. Color indicates data for different observation directions

say that there is no cloudiness effect on the estimated wind velocity and direction. The radiation scattered in the cloud layer and recorded in a certain direction results from mixing of radiation coming from different directions above the cloud layer. This can seriously distort wind data. In the same way, the observed temperature could be affected by the light scattering, but the recorded significant temperature decrease (the interval from $01: 30$ to $03: 30$ on January 27,2017 ) does not synchronize with the occurrence of cloudiness.

In addition, it should be noted that cloud scattering would lead to the observed increase in temperature since the emission of lines, shifted due to the presence of wind in different directions, "mix" in the clouds, thereby broadening the observed line.

In the immediate vicinity of the interferometer, there are a number of optical instruments detecting airglow. Next, we compare some observation results obtained with the interferometer to data acquired with an all-sky camera operating at a wavelength of 630.0 $\mathrm{nm}$, with a spectrometer recording 630.0 and 557.7 $\mathrm{nm}$ atomic oxygen intensities, and with an infrared spectrometer recording hydroxyl emission ((6-2) band, $834.0 \mathrm{~nm}$ ) in the mesopause region.

\section{NIGHT VARIATIONS OF INTENSITY AS OBTAINED WITH THE INTERFEROMETER, AND COMPARISON WITH SPECTROPHOTOMETER DATA}

Night variations in intensities of 630 and $557.7 \mathrm{~nm}$ atomic oxygen emissions are shown in Figure 7. The winter period is taken here because the time span of observations during this season is maximum. Intensity variations in the $630.0 \mathrm{~nm}$ line features an evening decrease associated with sunset, a slight midnight increase caused by an increase in the electron density at a height of emission [Akasofu, Chapman, 1974], and a morning increase due to sunrise. Vertical lines indicate the moments of sunset and sunrise for a height of $250 \mathrm{~km}$ above the interferometer and at the magnetoconjugate point. The dusk emission at $630 \mathrm{~nm}$ may be, to a greater extent, due to the photodissociation of molecular oxygen in the Schuman-Runge continuum; in the night period, the $630 \mathrm{~nm}$ emission is related to dissociative recombination with $\mathrm{O}_{2}^{+}, \mathrm{NO}^{+}$ions; and at pre-dawn hours, a notable contribution to the $630 \mathrm{~nm}$ emission can be made by photoelectrons from magnetoconjugate regions [Semenov, 1975; Krassovsky et al., 1976; Toroshelidze, 

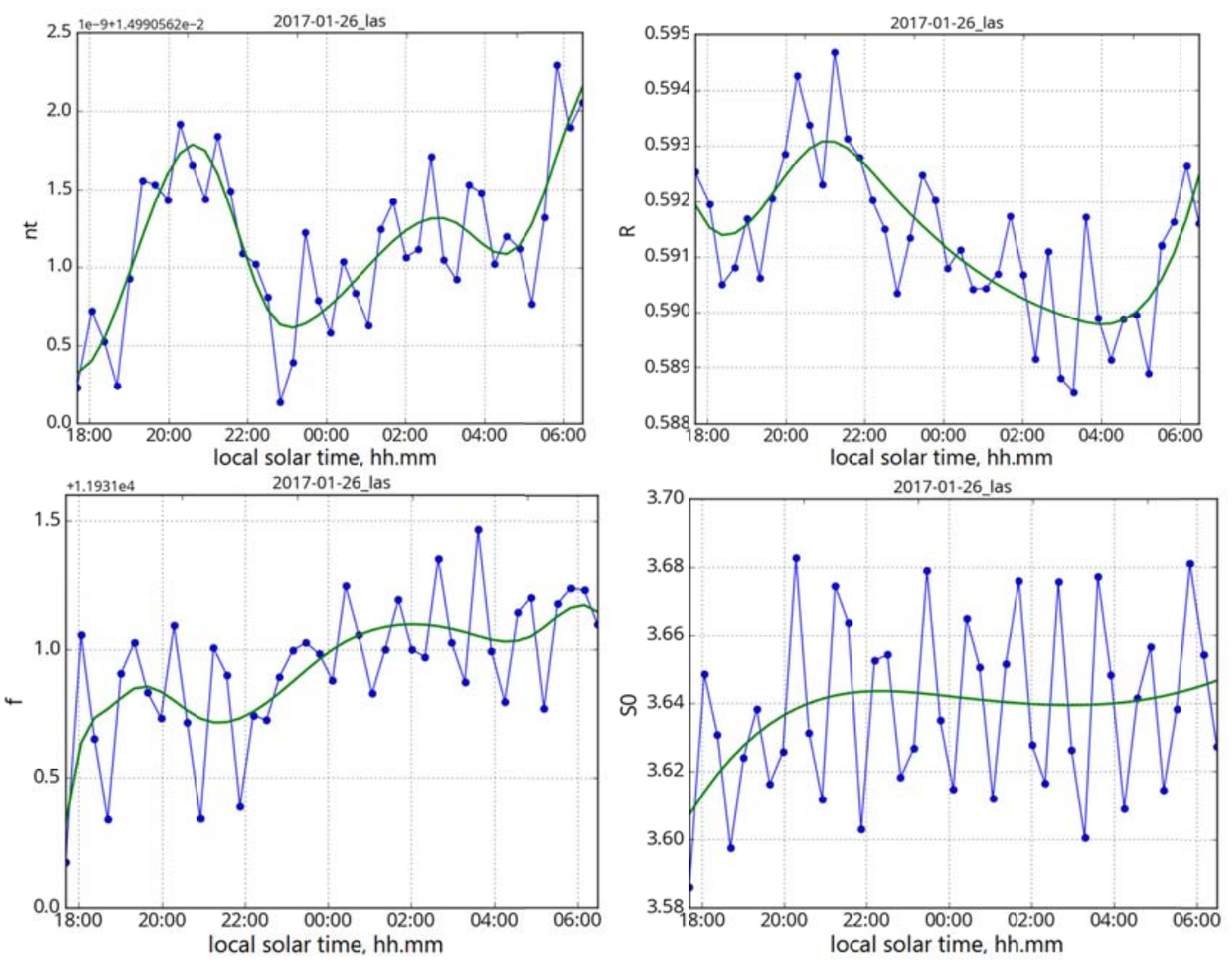

Figure 6. Product of the gap between the etalon and the refractive index (meters) (top left); reflection coefficient of the etalon's surfaces (top right); focal length (in CCD matrix pixels) (bottom left); width of the Gaussian kernel blurring the image (in matrix pixels) (bottom right). The green line indicates smoothed data used in the model of night-sky interferograms
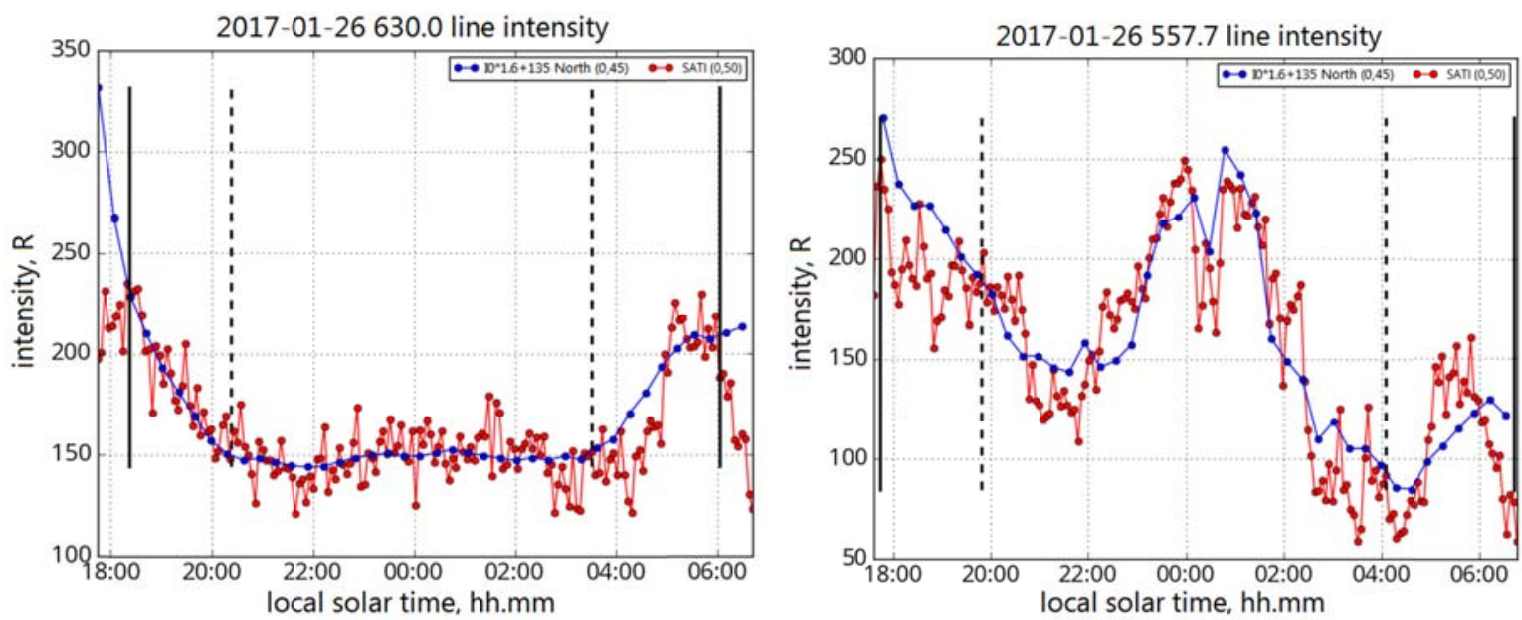

Figure 7. Night variations in the $630.0 \mathrm{~nm}$ emission intensity as derived from interferometer (blue) and spectrophotometer (red) data (left); night variations in the $557.7 \mathrm{~nm}$ emission intensity as derived from interferometer (blue) and spectrophotometer (red) data. Vertical lines are sunset and sunrise at 250 and $90 \mathrm{~km}$ above the interferometer (solid lines) and at the magnetoconjugated point (dashed lines)

1991, Kononov, Tashchilin, 2001]. The height of the maximum atomic oxygen emission of $557.7 \mathrm{~nm} \mathrm{(} \mathrm{97}$ $\mathrm{km})$ being much lower than that of $630 \mathrm{~nm}(\sim 250 \mathrm{~km})$, the times of local sunrise and sunset for $97 \mathrm{~km}$ are at the boundary of the observation interval. An observable characteristic feature of the night variation in the green line intensity in winter is the midnight maximum (Fish- kova, 1983), which arises from tidal activity. Figure 7 shows night variations in the 630 and $557.7 \mathrm{~nm}$ emission intensities as derived from data from the SATI-1 spectrophotometer [http://atmos.iszf.irk.ru/ru/groundbased/spectr] placed near the interferometer.

Interferometer data are taken for the region of the sky closest to the spectrophotometer field of view. 

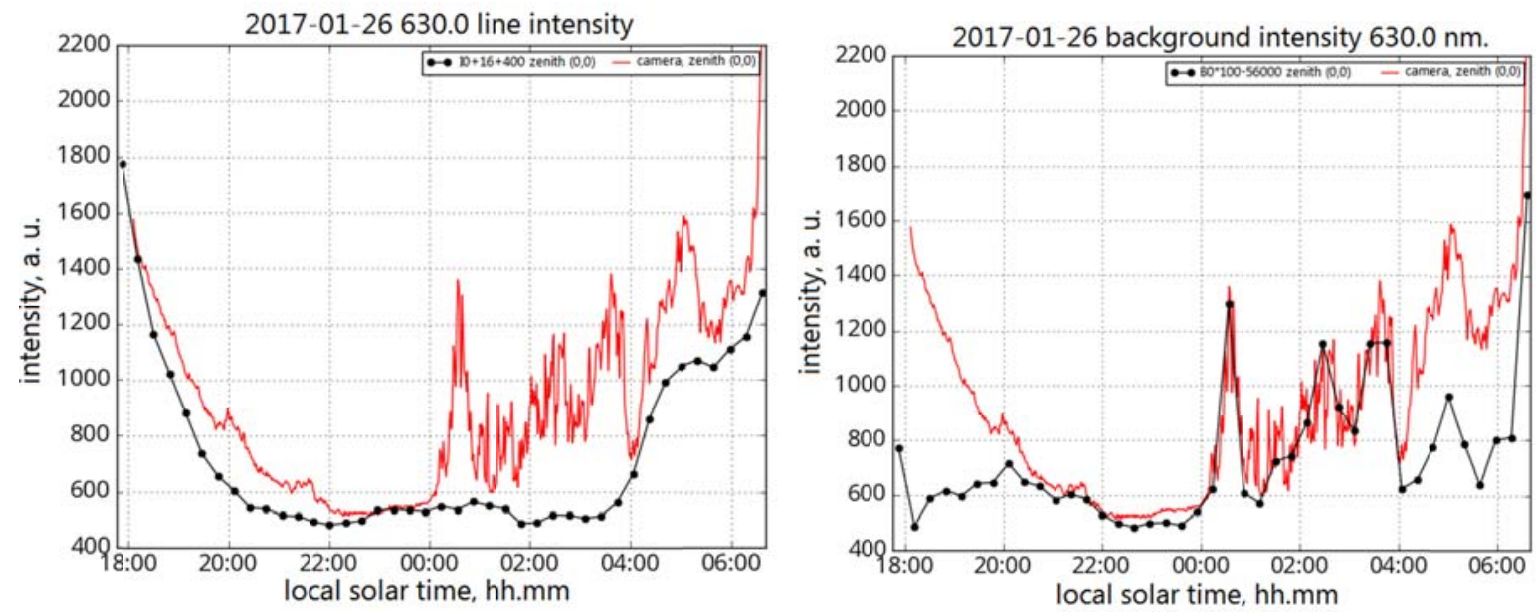

Figure 8. Night variations in the $630.0 \mathrm{~nm}$ line intensity as inferred from data obtained with the interferometer (black curve) and the all-sky camera (red curve); night variations in the background intensity for the $630.0 \mathrm{~nm}$ line as derived from data acquired with the interferometer (black curve) and integral intensity from the all-sky camera (red curve)

Night variations in the intensity of emissions derived from data obtained with various instruments are in good agreement; hence the nightglow intensity seems to be correctly represented in the interferogram model. At this stage, using spectrophotometer data, we can perform a simple calibration of the intensity recorded by the interferometer, assuming a linear relationship between the incoming light flux and the relative units of the interferometer intensity. For the green line, the correction coefficient is 4; for the red line, in addition to the multiplicative part, 1.6, there is an additive component equal to 135. This may be due to the presence of an additional background for the $630.0 \mathrm{~nm}$ line.

We have compared interferometer data to data obtained with a wide-angle camera equipped with a $630.0 \mathrm{~nm}$ filter [http://atmos.iszf.irk.ru/ru/ground-based/keo]. By comparison, in the images captured by the camera we select areas corresponding to the interferometer field of view. The behavior of the integrated intensity, recorded in these areas of the images, is shown in Figure 8. It is appropriate here to compare the results obtained with the camera to the behavior of not only the intensity of the red line, but also of the background, since the line and background intensities are not separated in the wide-angle camera in this case. The simultaneous observation with the camera and interferometer shows that a fairly good agreement of recorded intensities occurs in the absence of cloudiness (from the beginning of observations till midnight, see above), while in the presence of cloudiness (from midnight to the end of observations) a better agreement is observed between the integrated intensity recorded with the camera and the background recorded with the interferometer.

\section{NIGHT VARIATIONS \\ IN TEMPERATURE FROM \\ THE $630.0 \mathrm{~nm}$ LINE AND PROBLEM OF LOW TEMPERATURES}

Temperature variations as observed in the $630.0 \mathrm{~nm}$ line overnight are mainly as expected (Figure 9). There is an evening decrease and a morning increase in tem- perature caused by sunset and sunrise. As shown by neutral atmosphere models and observational data [Fisher et al., 2015], the maximum variation in the gaskinetic temperature at a height of around $250 \mathrm{~km}$ overnight is about $150 \mathrm{~K}$. The night variations in Doppler temperature $\mathrm{T}_{630} \sim 400-500 \mathrm{~K}$ observed in this case differ significantly from the NRLMSISE-00 model data. The values of $\mathrm{T}_{630}$ calculated from the empirical model [Shefov et al., 2007] for the niight of January 26, 2017, vary from $\sim 750 \mathrm{~K}$ at the beginning of the night and to $\sim 514 \mathrm{~K}$ in the pre-dawn hours around the local midnight, thus being also much higher than the temperature measured with the interferometer.

Note a rapid, about one hour, decrease in the observed Doppler temperature by about $200 \mathrm{~K}$ at 02:00. Such a significant temperature decrease cannot be caused by variations in parameters of the observation system since the behavior of the parameters $R$ and $S_{0}$ (Figures 5,6) does not correlate with the behavior of the observed temperature.

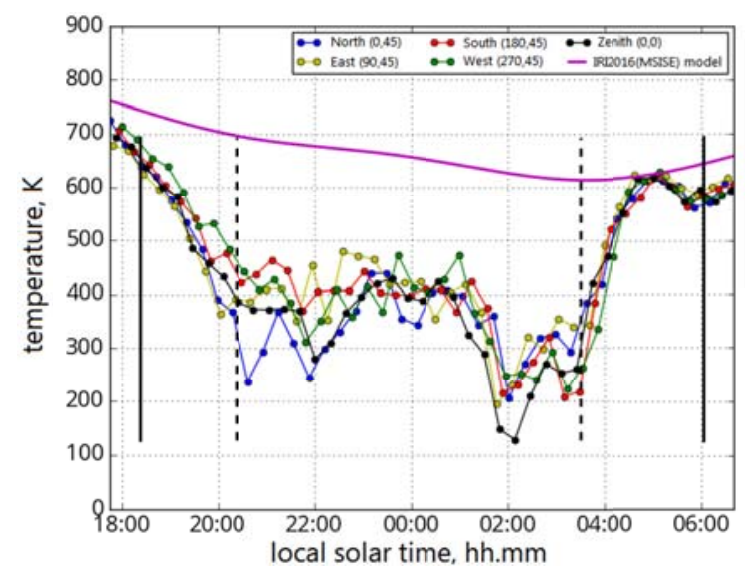

Figure 9. Night variations in temperature from the $630.0 \mathrm{~nm}$ line and NRLMSISE-00 data. Red, blue, green, and yellow curves are temperature values for different directions of observation; the purple curve indicates model data. Black vertical lines denote sunset and sunrise above the interferometer (solid) and at the magnetoconjugate point (dashed lines) 
Another factor capable of causing the temperature to fall to such abnormally low values may be the presence of a spurious signal, for example, the hydroxyl emission in the (9-3) band, which overlaps the wavelength of 630.0 nm. As shown in [Hernandez, 1974], such a hypothetical possibility exists.

It should be noted that Nakamura et al. [2017] report the results from determination of temperature from the $630 \mathrm{~nm}$ line and show that the temperature values obtained with the Fabry-Perot interferometer are periodically much lower than the atmosphere temperature at the height of the emission layer. The authors explain this problem of underestimation of the measured temperature by distortion of a signal, recorded with the interferometer, owing to the hydroxyl emission ((9-3) band) at a wavelength that almost coincides with the $630 \mathrm{~nm}$ atomic oxygen emission. Thus, other scientific teams also face the problem of systematic underestimation of temperature from interferometric measurements; therefore, it calls for further investigation. Some pilot studies of this problem can be carried out using instruments of GO Tory, which is equipped with a FabryPerot interferometer and a spectrometer, which allows us to examine variations in the hydroxyl emission intensity.

Let us examine in more detail several interferograms obtained during the night on January 26-27, 2017 at 18:00, 00:00, and 02:00 local solar time (Figure 10). As the 630.0 line intensity decreases, the second ring system becomes more pronounced in a one-dimensional interference pattern. It is not visible in two-dimensional interferograms due to a very noisy signal. If we construct model one-dimensional interferograms for wavelengths corresponding to the hydroxyl emission lines $P_{1}(2) 628.75, P_{2}(3) 629.79$, and $P_{1}(3) 630.7 \mathrm{~nm}$ [Hernandez, 1974] in one plot with experimental data, we can compare the second ring system with one of these lines. Figure 11 shows two interferograms for 18:00 and 02:00 local solar time. Experimental data, the most well-chosen model, and the model with wavelengths corresponding to hydroxyl emission and exact position of the oxygen line ${ }^{3} P_{2}-{ }^{1} D_{2} 630.0308 \mathrm{~nm}$ are plotted in different colors [Hernandez, 1974]. In this case, we need the model for the exact value of the oxygen line because there can be some constant systematic shift in the observation system at the accuracy level considered. Therefore, to identify the second line, we should shift all model curves of hydroxyl and oxygen ring systems to the right by the same amount to fit the model curves describing the oxygen ring system with observational data. Here the hydroxyl line $P_{1}(3)$ will coincide with the second ring system. According to [Hernandez, 1974], this line is the brightest in this triplet. This agrees with observations we perform. Thus, the data presented in Figure 11 suggest that with a significant decrease in the intensity of the oxygen line ${ }^{3} P_{2}-$ ${ }^{1} D_{2}$, the hydroxyl line $P_{1}(3)$ begins to resolve, but due to minimization the model still describes the brightest oxygen line. Hence, the temperature derived from minimization is in fact the temperature obtained from observation of the $630.0 \mathrm{~nm}$ line.
As already noted, the presence of cloudiness can distort parameters of observed optical radiation and thus can distort estimated wind and temperature characteristics. A considerable temperature decrease, by more than $100 \mathrm{~K}$, occurred almost immediately after sunset, and an additional decrease to $\sim 150 \mathrm{~K}$ began at 02:00. This does not correlate with the observed cloud behavior. Moreover, the lowest temperature at this night was recorded at the zenith, while the maximum background intensity hypothetically capable of distorting the results was observed westward.

Figure 12 shows variations in the intensity of the hydroxyl emission arising at mesopause heights ((62) band, $\sim 87 \mathrm{~km})$, and in ionospheric parameters: electron density at the ionization maximum $N_{\mathrm{m}}$ F2 and the height of the ionization maximum $H_{\mathrm{m}} \mathrm{F} 2$ versus direction-averaged intensity and Doppler temperature for the $630.0 \mathrm{~nm}$ line. The observations were made with an infrared spectrometer located in the immediate vicinity [Medvedeva et al., 2012] and with a DPS-4 ionosonde. The data are given for January 26,2017 . We can say that the behavior of the $630 \mathrm{~nm}$ line intensity correlates with the Doppler temperature behavior. This is especially noticeable during temperature anomaly. The behavior of the hydroxyl emission intensity anticorrelates with the behavior of the observed temperature, but this anticorrelation can in some cases be delayed for some time, and in some cases may be absent (Figure 13), despite the abnormally low temperature. It should be noted that the temperature behavior during the anomaly on January 26, 2017 is in good agreement with the behavior of the charged component of the upper atmosphere (Figure 12, right).

This event is of special interest and will be considered in detail in follow-up studies.

\section{NIGHT VARIATIONS IN TEMPERATURE FROM THE $557.7 \mathrm{~nm}$ LINE. CALIBRATION PROBLEM. COMPARING WITH DATA ON ROTATIONAL TEMPERATURE OF HYDROXYL MOLECULES}

It is unreasonable to use a helium-neon laser for calibration with the $557.7 \mathrm{~nm}$ light filter because a signal in this case is extremely weak. The model parameter which greatly depends on emission wavelength and is of key importance in determining temperature is the reflection coefficient $R$. To work in the green spectral region, we received a laboratory-measured wavelength dependence of the reflection coefficient of the etalon's surfacesfrom manufacturers of the interferometer. This dependence was used to scale this parameter in the instrumental function while minimizing for data obtained at a wavelength of $557.7 \mathrm{~nm}$. Night variations in temperature as observed in the green line of atomic oxygen for two different nights are shown in Figure 14. The absolute temperature as derived from the interferometer 

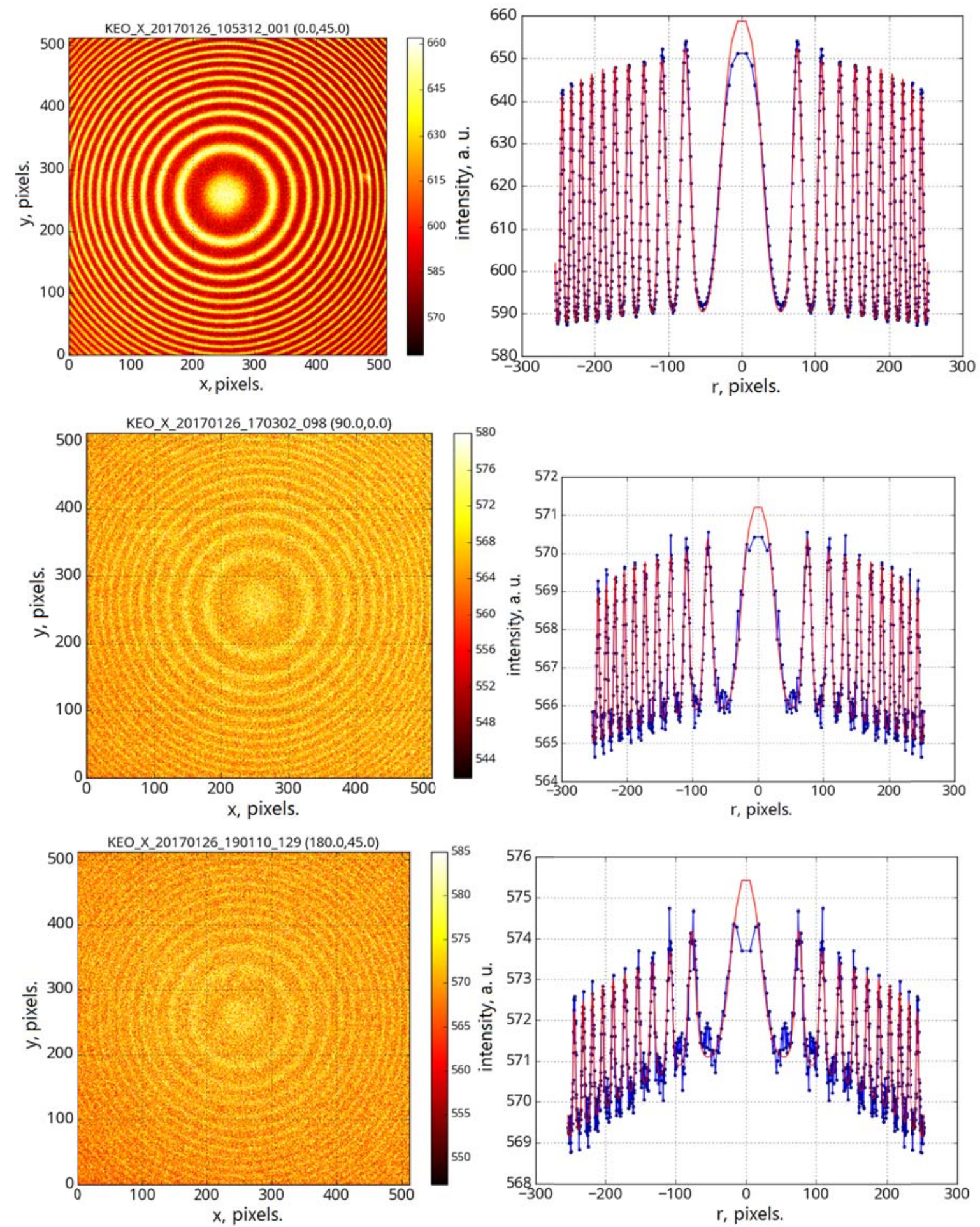

Figure 10. Two-dimensional interferograms obtained at 18:00, 00:00, and 02:00 local solar time (left, from top to bottom). One-dimensional interferograms obtained in the same periods (right, from top to bottom). Blue curves indicate observational data; the red curve, the model selected from the observational data 

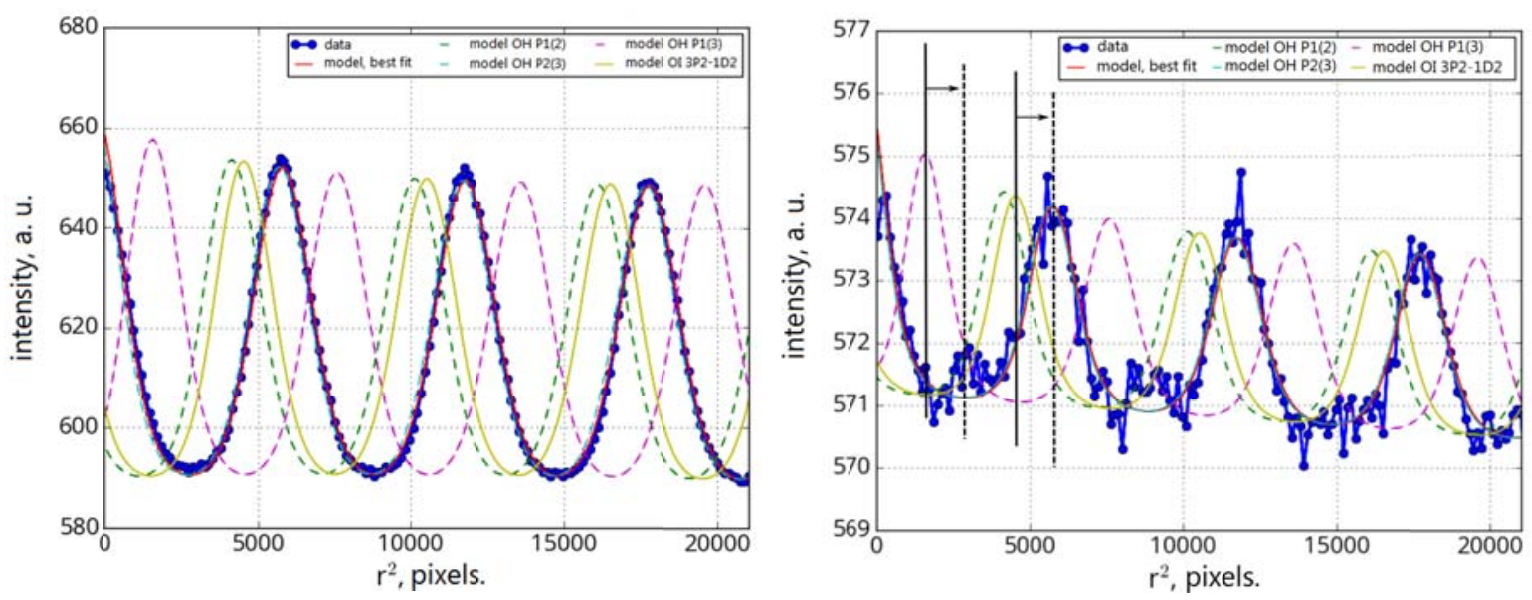

Figure 11. A part of the interferogram for 18:00 local solar time (left). The blue curve indicates observational data; the red one is the model selected from the observational data, the yellow curve is the model selected from the observational data, but with a wavelength of $630.0308 \mathrm{~nm}$. Dashed curves are models selected from the observational data, but with wavelengths corresponding to the three nearest hydroxyl emission lines as derived from data obtained in [Hernandez, 1974]. Arrows and vertical lines show the direction and value of the shift needed to account for the systematic bias in the observation results
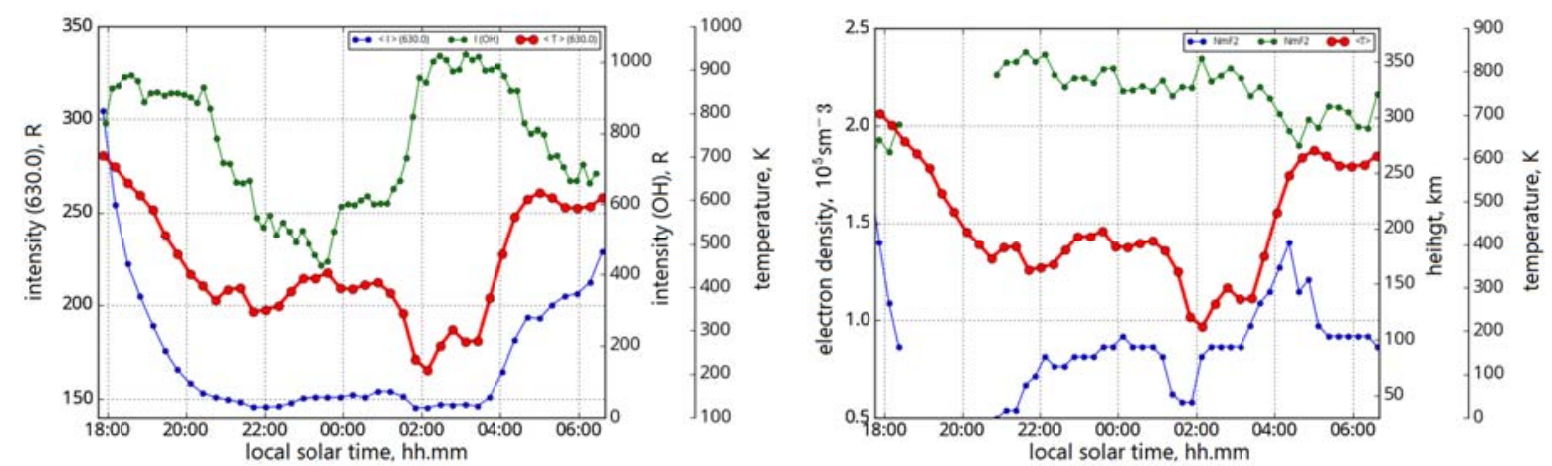

Figure 12. Behavior of Doppler temperature (left, red curve) and intensity (left, blue curve) from the $630.0 \mathrm{~nm}$ line for the night of January 26-27, 2017. Green color marks variations in the hydroxyl emission intensity, the (6-2) band. Temperature variations (right, red curve) from the $630.0 \mathrm{~nm}$ line and variations in ionospheric parameters: the height of the F2-layer maximum (green curve) and electron density at the F2-layer maximum (blue curve)

data turned out to be much higher than expected due to the insufficient accuracy of $R$, therefore the Figures use a scaled value.

To approximately calibrate interferometric temperature measurements, we can use data on rotational temperature of hydroxyl molecule obtained from spectral observations of $\mathrm{OH}$ molecule emission $((6-2)$ band, 834

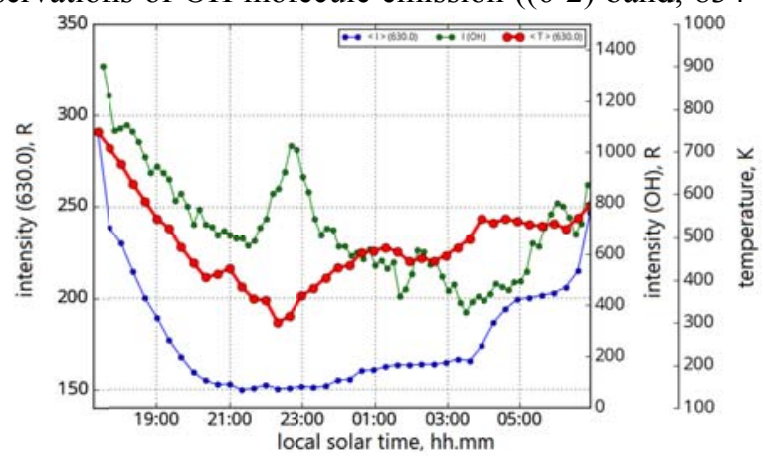

$\mathrm{nm})$ with the infrared spectrometer located in the immediate vicinity [Medvedeva et al. 2012]. The hydroxyl emission layer intensity is maximum at a height of $\sim 87$ $\mathrm{km}$ and is $\sim 9 \mathrm{~km}$ thick; the rotational temperature of the $\mathrm{OH}$ molecule corresponds to the atmospheric temperature at the emission height [Shefov et al., 2006].

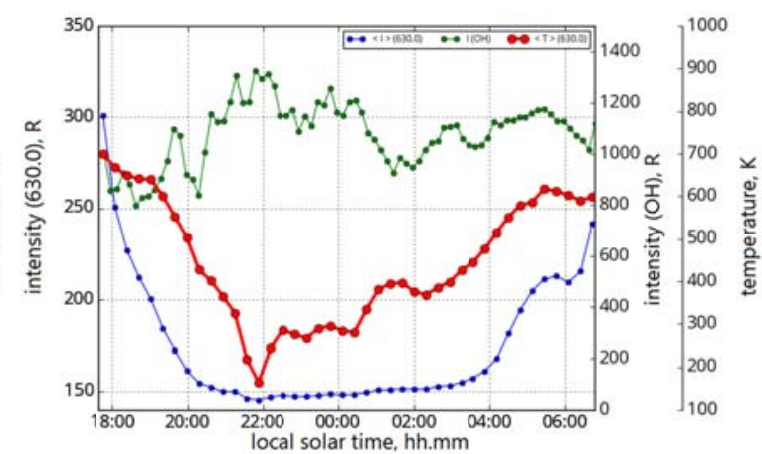

Figure 13. Variations in the Doppler temperature (red curves) and intensity (blue curves) from the $630.0 \mathrm{~nm}$ line. Green curves are hydroxyl emission intensity variations (6-2). Variations for the night of January 2, 2017, when there was delayed anticorrelation between the Doppler temperature of the $630 \mathrm{~nm}$ line and the hydroxyl emission intensity (left). Variations for the night of January 21, 2017, when there was no anticorrelation between the Doppler temperature of the $630 \mathrm{~nm}$ line and the hydroxyl emission intensity (6-2) 

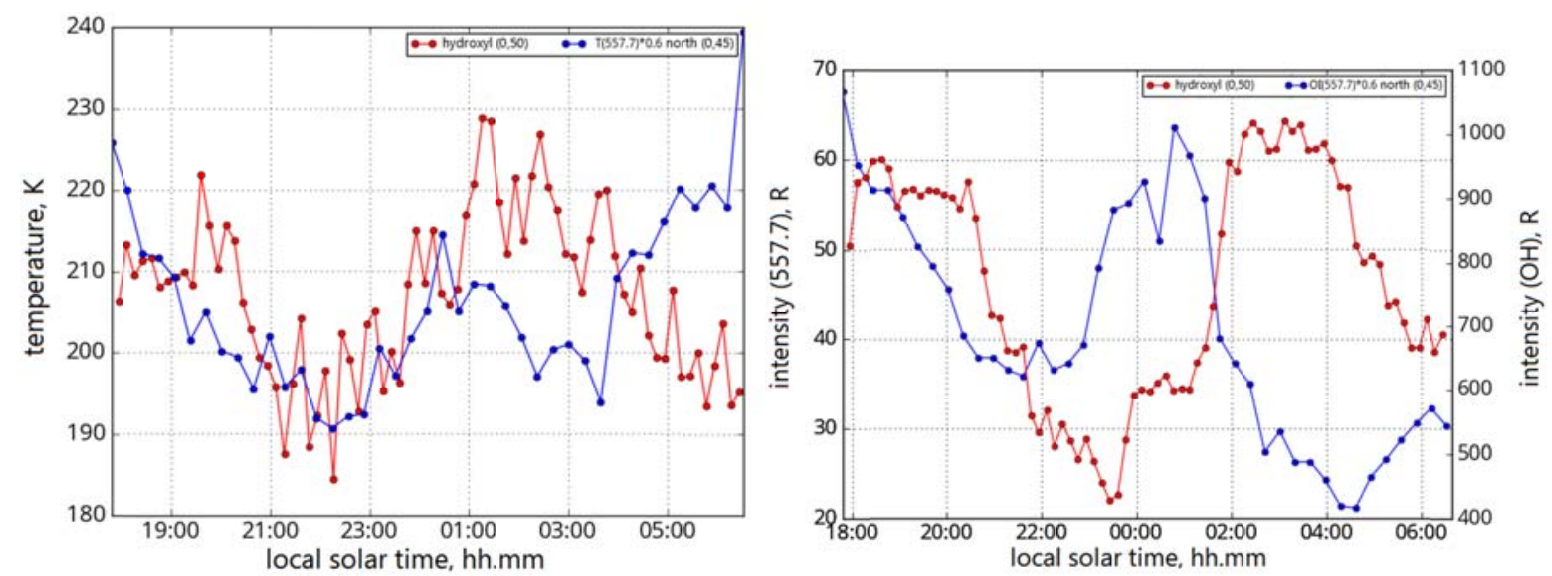

Figure 14. Night variations in temperature obtained from observations of $\mathrm{OH}$ emission (6-2) (red curve) and green oxygen line (blue curve) (left). Night variations in $\mathrm{OH}$ emission intensity (6-2) (red curve) and green oxygen line (blue curve) for January 26, 2017 (right)

Since the maximum atomic oxygen emission at $557.7 \mathrm{~nm}$ $(\sim 97 \mathrm{~km})$ is relatively close to the height of the maximum hydroxyl emission $(\sim 87 \mathrm{~km})$, we can compare the temperature values and, in some approximation, determine the correction coefficient for the temperature determined from interferometric observations of the $557.7 \mathrm{~nm}$ emission. To fit the observed temperatures, we use a correction coefficient of 0.6 for the interferometer data. It is evident that for two nights the use of this coefficient gives good agreement of temperature variations approximately till 00:00, after which the temperature values obtained from observations of hydroxyl and atomic oxygen emissions begin to differ. A possible reason for this may be a change in wind conditions at $90-100 \mathrm{~km}$. As shown in Figure 15, it is at this time that the wind parameters, obtained using the green oxygen line, change.

\section{NIGHT VARIATIONS IN WIND AS DERIVED FROM 630.0 AND 557.7 nm EMISSION DATA}

Wind direction and velocity determined from both

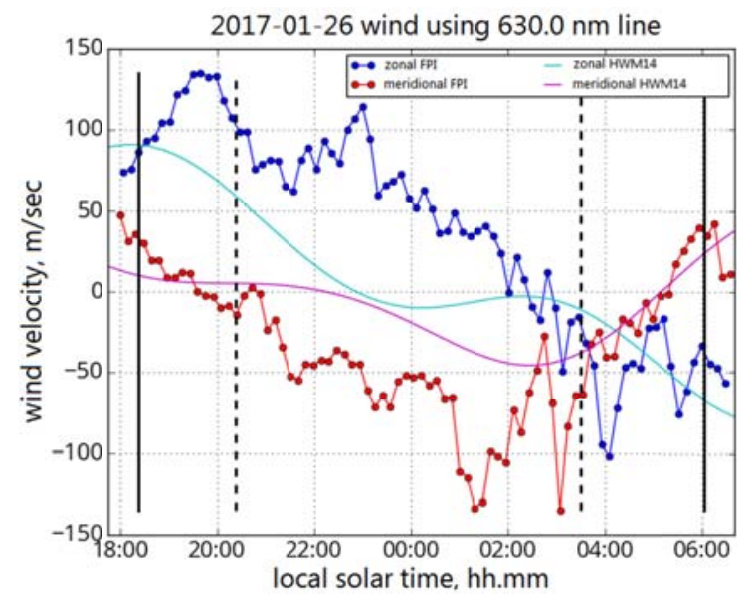

557.7 and $630.0 \mathrm{~nm}$ lines are shown in Figure 15. The Figure also presents wind variations obtained generally consistent with model data.

Absolute wind velocity and characteristic times reflecting maximum and minimum daily wind values roughly coincide. The differences seem to arise from peculiarities of upper atmosphere conditions and conditions of observations on a particular day. Wave activity, specific geomagnetic conditions, transfer of energy stored in the troposphere from the bottom up, clouds, and other factors, ignored in the HWM14 model, can significantly distort the wind pattern. Furthermore, the model HWM14 disregards solar activity, hence there are significant differences in wind amplitude. In order to correctly compare wind characteristics obtained with the interferometer to HWM14 model data, follow-up studies should average observed characteristics or consider specific conditions of the upper atmosphere in input parameters of the model.

Figure 15. Night variations in zonal and meridional winds (left), obtained from observations of the red oxygen line (blue and red curves) on January 26, 2017. On the right is the same for the green oxygen line. Blue and violet curves are zonal and meridional winds from the HWM14 model 


\section{CONCLUSIONS}

A new instrument designed to determine spectral characteristics of optical airglow at middle latitudes has been put into operation. We have carried out observations of characteristics of the spectral oxygen lines with wavelengths of 557.7 and $630 \mathrm{~nm}$. Using the obtained characteristics, we have determined the behavior of line and background intensities overnight, as well as the behavior of temperature and wind velocity for different heights in Earth's atmosphere. We have compared the results to data obtained with other instruments and to model outputs.

We have analyzed some factors and geophysical conditions that potentially affect the accuracy of measurements. In particular, we have shown that for the night of January 26, 2017 considered, the hydroxyl emission ((9-3) band), whose lines fall into the passband of the $630 \mathrm{~nm}$ interference filter, could not have a significant effect on the determination of the temperature $\mathrm{T}_{630}$. During some nights, there is characteristic anticorrelation between the behavior of hydroxyl emission intensity ((6-2) band) and temperature $\mathrm{T}_{630}$, which could be an additional factor exhibiting the effect of hydroxyl signal on estimated temperature. However, this anticorrelation is often delayed (of order of half an hour), and in some cases, at a significant decrease in $\mathrm{T}_{630}$, there is no anticorrelation. These facts may suggest that the relationship between the observed temperature $T_{630}$ and the hydroxyl emission intensity is formed by processes occurring in the upper atmosphere, and is not an error in observation or processing of the results.

We have noted the nature and degree of influence of the background emission caused by atmospheric scattered radiation background from external natural sources. We have compared 557.7 and $630 \mathrm{~nm}$ emission intensities, temperature $(557.7 \mathrm{~nm}$ emission) with data from the all-sky camera in the $630 \mathrm{~nm}$ emission, the SATI-1 spectrometer (557.7 and $630 \mathrm{~nm}$ emission spectrometer), and the infrared spectrometer (rotational $\mathrm{OH}$ temperature ((6-2) band). We have calculated and reported model values (NRLMSISE-00, HWM14) of kinetic temperatures and winds for heights of the maximum 557.7 and $630 \mathrm{~nm}$ emission at the site of the Fabry-Perot interferometer observation. A fairly good agreement has been obtained for model and experimentally observed values of wind velocity, whereas temperatures $\mathrm{T}_{630}$ in some intervals significantly differ from model values. The closest agreement between temperatures is observed in the pre-dusk hours. The most acute and still unresolved issue in these results is the observed extremely low $(\sim 200 \mathrm{~K})$ temperatures (in the period 01:30-03:30 LT), determined from the $630 \mathrm{~nm}$ emission. In this interval, a sufficiently high correlation of $\mathrm{T}_{630}$ with $f_{0} \mathrm{~F} 2$, related to the $630 \mathrm{~nm}$ line emission region, does not allow us to unambiguously associate this result with the inaccuracy in the interferometric technique of determining $\mathrm{T}_{630}$ or with the influence of meteorological conditions; it requires additional experimental and theoretical studies and, possibly, refinement of the technique for processing interferometric observations.

Note that a possible explanation for the observed extremely low temperatures, comparable to the tempera- tures at heights of the upper mesosphere-lower thermosphere in the $557.7 \mathrm{~nm}$ emission region $(\sim 85-115 \mathrm{~km})$, could be one of the known channels of population of the $1^{\mathrm{D}}$ atomic oxygen level associated with the $557.7 \mathrm{~nm}$ emission at the transition from ${ }^{1} \mathrm{~S}$ to ${ }^{1} \mathrm{D}$. In this case, each emission of $557.7 \mathrm{~nm}$ photon produces one excited $\mathrm{O}\left({ }^{1} \mathrm{D}\right)$ atom With hypothetical extreme decreases in the $630 \mathrm{~nm}$ emission intensity from the ionospheric F2 region formed by dissociative recombination, a certain contribution to the height-integral $630 \mathrm{~nm}$ emission intensity (and hence to the temperature) could also be made by the emission from this wavelength from the $557.7 \mathrm{~nm}$ emission height. However, it is generally accepted that, because of the long lifetime of the metastable level ${ }^{1} \mathrm{D}$ and high rates of its collisional deactivation at these heights, there is no $630 \mathrm{~nm}$ emission at the upper mesosphere-lower thermosphere heights. The relationship between kinetic temperatures obtained with atmospheric models and Doppler temperatures obtained from interferometric observations in the $630 \mathrm{~nm}$ emission will be examined more thoroughly in follow-up studies.

The comparison between the temperature obtained from observations of the $\mathrm{OH}$ emission (6-2) and that obtained from observations of the $557.7 \mathrm{~nm}$ oxygen line shows a significant discrepancy between these characteristics, despite the fact that the emission sources are vertically spaced by about $10 \mathrm{~km}$. So, it is unacceptable to use hydroxyl emission to calibrate the interferometer that records emission at $557.7 \mathrm{~nm}$. Therefore, for the $557.7 \mathrm{~nm}$ line, we should search for additional means of calibration of the instrument. In this case, it may be reasonable to use a low-pressure mercury lamp, which has a line in the green spectral region, as a source of line optical radiation.

In general, the results obtained with the Fabry-Perot interferometer designed to observe the airglow in two atomic oxygen lines of 557.7 and $630.0 \mathrm{~nm}$ demonstrate validity of this instrument and its quite high potential for follow-up studies of thermodynamic mesospherelower thermosphere characteristics.

We have used data from the Fabry-Perot interferometer included in the optical complex of the Angara Multiaccess Center.

The work was financially supported by the project "Study of dynamic processes in the neutral atmosphereionosphere-magnetosphere system" (unique number 03442014-0006) and grant No. NSH-6894.2016.5 of the President of the Russian Federation for State Support of Leading Scientific Schools.

\section{REFERENCES}

Akasofu S.I., Chepmen S. Solar-Terrestrial Physics. Part 1. Moscow, Mir Publ., 1974. 384 p. (In Russian).

Anderson C., Conde M., Dyson P., Davies T., Kosch M.J. Thermospheric winds and temperatures above Mawson, Antarctica observed with an all-sky imaging Fabry-Perot spectrometer. Ann. Geophys. 2009, vol. 27, pp. 2225-2235. DOI: 10.5194/angeo-27-2225-2009.

Born M., Wolf E. Osnovy optiki [Principles of Optics. $2^{\text {nd }}$ Ed.]. Moscow: Nauka Publ., 1973, pp. 297-313. (In Russian).

Coelho L.P. Mahotas. Open source software for scriptable computer vision. J. Open Res. Software. 2013, 1:e3. DOI: http://dx.doi.org/10.5334/jors.ac 
Drob D.P., Emmert J.T., Meriwether J.W., Makela J.J, Doornbos E.N, Conde M., Hernandez G., Noto J., Zawdie K.A., McDonald S.E., Huba J.D., Klenzing J.H. An update to the Horizontal Wind Model (HWM): The quiet time thermosphere. Earth and Space Sci. 2015, vol. 2, pp. 301-319. DOI: 10.1002/2014EA000089.

Fisher D.J., Makela J.J., Meriwether J.W., Fisher D.J., Makela J.J., Meriwether J.W., Buriti R.A., Benkhaldoun Z., Kaab M., Lagheryeb A. Climatologies of nighttime thermospheric winds and temperatures from Fabry-Perot interferometer measurements: From solar minimum to solar maximum. J. Geophys. Res. Space Phys. 2015, vol. 120, pp. 66796693. DOI: 10.1002/2015JA021170.

Fishkova L.M. Nochnoe izluchenie sredneshirotnoi verkhnei atmosfery Zemli [Night Emission of the Earth's MidLatitude Upper Atmosphere]. Tbilisi: Metsniereba Publ., 1983, 272 p. (In Russian).

Harding B.J., Gehrels T.W., Makela J.J. Nonlinear regression method for estimating neutral wind and temperature from Fabry-Perot interferometer data. App. Optics. 2014, vol. 53, pp. 666-673. DOI: 10.1364/AO.53.000666.

Hernandez G. Contamination of the OI ( ${ }^{3} \mathrm{P} 2-^{-} \mathrm{D}$ 2) emission line by the (9-3) band of $\mathrm{OH} \mathrm{X}^{2} \mathrm{II}$ in high-resolution measurements of the night sky. J. Geophys. Res. 1974, vol. 79, no. 7, pp. 1119-1123. DOI: 10.1029/JA079i007p01119.

Ignatyev V.M., Yugov V.A. Interferometriya krupnomasshtabnoi dinamiki vysokoshirotnoi termosfery [Interferometry of Large-Scale Dynamics of High-Latitude Thermosphere]. Yakutsk Scientific Center. Yakutsk, 1995, 208 p. (In Russian).

Ignatyev V.M., Nikolashkin S.V., Yugov V.A., Asksentyev A.G., Ammosov P.P. Fabry-Perot high-transmission spectrometer. Pribory i tekhnika eksperimenta [Instruments and Experimental Techniques]. 1998, no. 4, pp. 107-110. (In Russian).

Kononov R.A., Tashchilin A.V. Influence of seasonal and cyclic variations of thermospheric parameters on the night intensity of the red line of atomic oxygen. Atmospheric and Oceanic Optics. 2001, vol. 14, no. 10. pp. 979-982.

Krassovsky V.I., Semenov A.I., Shefov N.N. Predawn emission at $6300 \AA$ and super-thermal ions from conjugate points. J. Atm. Terr. Phys. 1976, vol. 38, no. 9-10, pp. 9991001.

Landau L.D., Lifshits E.M. Teoriya polya [Theory of Fields. $7^{\text {th }}$ Ed.]. Moscow: Nauka Publ., 1988, pp. 158-159. (In Russian).

Makela J.J., Meriwether J.W., Huang Y., Sherwood P.J. Simulation and analysis of a multi-order imaging Fabry-Perot interferometer for the study of thermospheric winds and temperatures. Appl. Optics. 2011, vol. 50, pp. 4403-4416. DOI: 10.1364/ AO.50.004403.

Marquardt D.W. An algorithm for least-squares estimation of nonlinear parameters. J. Soc. Industrial and Applied Mathematics. 1963, vol. 11, no. 2, pp. 431-441. DOI: $10.1137 / 0111030$.

Medvedeva I.V., Semenov A.I., Perminov V.I., Tatarnikov A.V., Beletsky A.B. Comparative analysis between data of ground-based measurements of mesopause temperature at midlatitudes and satellite data MLS Aura, v3.3. Sovremennye problem distantsionnogo zondirovaniya Zemli iz kosmosa [Current Problems in Remote Sensing of the Earth from Space]. 2012, vol. 9, no. 4, pp. 133-139. (In Russian).

Nakamura Y., Shiokawa K., Otsuka Y., Oyama S., Nozawa S., Komolmis T., Komonjida S., Neudegg D., Yuile C., Meriwether J., Shinagawa H., Jin H. Measurement of thermospheric temperatures using OMTI Fabry-Perot interferometers with 70-mm etalon. Earth, Planets and Space. 2017, vol. 69, iss. 1, article id.57. DOI: 10.1186/s40623-017-0643-1.
Newville M., Stensitzki T., Allen D.B., Ingargiola A. LMFIT: Non-Linear Least-Square Minimization and Curve-Fitting for Python [Data set]. Zenodo, 2014. URL: http://doi.org/ 10.5281/zenodo.11813 (accessed Yuly 14, 2017).

Picone J.M., Hedin A.E., Drob D.P., Aikin A.C. NRLMSISE-00 empirical model of the atmosphere: Statistical comparisons and scientific issues. J. Geophys. Res. 2002, vol. 107, no. A12, p. 1468. DOI: 10.1029/2002JA009430.

Semenov A.I. Predawn temperature and intensity variations of $6300 \AA$ emission. Astronomicheskii tsirkulyar [Astronomical Circular]. 1975, no. 882, pp. 6-7. (In Russian).

Shefov N.N., Semenov A.I., Khomich V.Yu. Izluchenie verkhnei atmosfery — indicator ee struktury I dinamiki [Upper Atmosphere Emission as Indicator of Its Structure and Dynamics]. Moscow: Geos, 2006, 741 p. (In Russian).

Shefov N.N., Semenov A.I., Yurchenko O.T., Sushkov A.V. Empirical model of variations of $630.0 \mathrm{~nm}$ atomic oxygen emission. 2. Temperature. Geomagnetizm i aeronomiya [Geomagnetiosm and Aeronomy]. 2007, vol. 47, no. 5, pp. 692-701. (In Russian).

Shiokawa K., Otsuka Y., Oyama S. Development of lowcost sky-scanning Fabry-Perot interferometers for airglow and auroral studies. Earth, Planets and Space. 2012, vol. 64, p. 1033. DOI: $10.5047 /$ eps.2012.05.004.

Toroshelidze T.I. Analiz problem aeronomii po izlucheniyu verkhnei atmosfery [Analysis of Aeronomy Problems from Upper Atmosphere Emission]. Tbilisi: Metsniereba Publ., 1991, 216 p. (In Russian).

van Rossum G. Python Tutorial, Technical Report CSR9526. Centrum voor Wiskunde en Informatica (CWI). Amsterdam, May 1995.

Wu Q., Gablehouse R.D., Solomon S.C., Killeen T.L., Chiao-Yao She. A new NCAR Fabry-Perot interferometer for upper atmospheric research. Proc. SPIE, 2004, vol. 5660, pp. 218-227.

URL: http://atmos.iszf.irk.ru/ru/ground-based/spectr (accessed Yuly 14, 2017).

URL: http://atmos.iszf.irk.ru/ru/ground-based/keo (accessed Yuly 14, 2017).

\section{How to cite this article}

Vasilyev R.V., Artamonov M.F., Beletsky A.B., Zherebtsov G.A., Medvedeva I.V., Mikhalev A.V., Sirenova T.E. Registering upper atmosphere parameters in East Siberia with Fabry-Perot interferometer KEO Scientific “Arinae". Solar-Terrestrial Physics. 2017. Vol. 3, iss. 3. P. 61-75. DOI: $10.12737 /$ stp-33201707 